of the form $2^{n} x_{1}$ or $x_{1} / 2^{n}$. Also for these values, we see from (38) that $S(x)=j \sinh m x$, where $j$ is plus or minus one, and must be the same for all such values of $x$, since (28) shows that

$$
S(2 x)=2 S(x) C(x) \text {. }
$$

Finally, since (28) and (37) are the addition formulas for $\sinh m x$ and $\cosh m x$, we see that these functions agree with $S(x)$ (to within a sign) and $C(x)$ for all multiples of $x_{1}$ whose fractional parts are terminating decimals in the binary scale, and hence, since all the functions concerned are continuous, at all points. Thus under I, II and III $(c)$, the family is necessarily $A \sinh m x+B \cosh m x$.

7. Conclusions. In conclusion, we notice that since I and II alone must determine one of the three types of families discussed, we may use any characteristic property of the types in place of III. Thus, we might replace III $(a)$ by the assumption "Some member of the family vanishes twice," or "Every member of the family is bounded." This last statement may be extended so as to give an alternative form of the assumption III, in terms of bounded, instead of nonvanishing functions. That is, III $(a),(b)$ and $(c)$ above may be replaced by the following postulates:

III $\left(a^{\prime}\right)$. There exist two linearly independent members of the family which are bounded.

III $\left(b^{\prime}\right)$. There exists one member of the family which is bounded, and all other bounded members of the family are linearly dependent on this one.

III $\left(c^{\prime}\right)$. No member of the family is bounded.

HARVARD UNIVERSITY

\title{
GROUPS IN WHICH THE NUMBER OF OPERATORS IN A SET OF CONJUGATES IS EQUAL TO THE ORDER OF THE COMMUTATOR SUBGROUP*
}

BY G. A. MILLER

1. Introduction. From the fact that the commutator quotient group is abelian, it results directly that there is no

\footnotetext{
* Presented to the Society, Sept. 7, 1922.
} 
group in which the number of operators in a complete set of conjugates is larger than the order of the commutator subgroup. Hence the groups which satisfy the condition noted in the heading of this paper contain the largest possible number of operators in a complete set of conjugates. They evidently include all the abelian groups as a special case, since the commutator subgroup of every abelian group is the identity. On the other hand, they do not include any simple group of composite order, since such a group is identical with its commutator subgroup.

Each of the groups which satisfy the condition noted in the heading of this paper must involve a co-set as regards its commutator subgroup such that all the operators of this coset have the same order. Moreover, whenever the commutator subgroup contains more than one Sylow subgroup of a given order, it must transform these Sylow subgroups according to a simply transitive group. If this were not the case, all the operators in a co-set with respect to the commutator subgroup could not transform these Sylow subgroups according to substitutions of the same degree. This common degree would clearly have to be equal to the number of the Sylow subgroups of the same order diminished by one for every set of conjugate Sylow subgroups found in the commutator subgroup.

From the preceding paragraph it results that if a group $G$ contains a set of conjugate operators whose number is equal to the order of the commutator subgroup of $G$, then this subgroup must satisfy certain conditions. In fact, some groups cannot be the commutator subgroup of any group whatsoever. For instance, it is easy to prove that no dihedral group whose order exceeds 4 can be a commutator subgroup. In fact, none of the non-invariant operators of order 2 contained in such a group could be a commutator since the product of such an operator and some other operator could not transform the generators of the characteristic cyclic subgroup into the same power of themselves as the latter operator. It is also easy to prove that no symmetric group whose order exceeds 2 can be the commutator subgroup of a group. 
In fact, if a symmetric group were the commutator subgroup of a group, an operator $t$ outside of this symmetric group would have to be transformed into itself multiplied by an operator represented by a negative substitution of the symmetric group. It is evident that this product could not correspond to $t$ in an automorphism of the group when the order of the symmetric group is neither 2 nor 720 . In the special case when the order of the symmetric group is 720 this group admits 720 outer isomorphisms. If $t$ transformed the symmetric group according to an outer isomorphism it results again that the given product could not correspond to $t$ in an automorphism since this product would transform the operators of order 5 in the symmetric group into a different power than $t$ does. While no symmetric group whose order exceeds 2 can be the commutator subgroup of any group whatever, every alternating group is the commutator subgroup of the corresponding symmetric group. An alternating group whose order exceeds 3 cannot, however, be the commutator subgroup of a group in which the number of operators in a set of conjugates is equal to the order of the commutator subgroup. It is interesting to inquire what groups can be commutator subgroups of such groups. In the next section we shall prove that every abelian group has this property.

2. Abelian Commutator Subgroups. Let $H$ be an arbitrary abelian group of order $h$. It is not difficult to construct a group $G$ which has $H$ for its commutator subgroup and involves a set of $h$ conjugate operators. When $h$ is odd such a $G$ can evidently be found by adjoining to $H$ an operator of order 2 which transforms every operator of $H$ into its inverse. The group thus obtained is clearly the generalized dihedral group of order $2 h$.

The generalized dihedral group obtained by extending $H$ by means of an operator of order 2 which transforms every operator of $H$ into its inverse has evidently always for its commutator subgroup the group generated by the squares of the operators found in $H$, and the number of operators in each of its complete sets of conjugate non-invariant operators 
of order 2 is equal to the order of this commutator subgroup. When $h$ is even we can therefore construct the required $G$ by first extending $H$ by operators which are commutative with every operator of $H$ and have for their squares the various operators in a set of independent generators of $H$ whose orders are of the form $2^{\alpha}$. By extending the abelian group $H$ thus obtained by means of an operator of order 2 which transforms each of its operators into its inverse we obtain a group which has $H$ for its commutator subgroup and in which the number of operators in the complete sets of conjugate noninvariant operators of order 2 is equal to $h$.

The order of the $G$ thus constructed is equal to the order of $H$ multiplied by $2^{\beta+1}$, where $\beta$ is equal to the number of the invariants of $H$ which are of the form $2^{\alpha}$. All the operators whose orders exceed 2 in this $G$ are conjugate in pairs, while the number of the complete sets of $h$ conjugates is $2^{\beta}$. It is easy to construct by other methods groups which satisfy the given conditions. For instance, if we extend the generalized dihedral group of order $2 h$, obtained by extending $H$ by means of an operator of order 2 which transforms every operator of $H$ into its inverse, by means of the operators in its group of isomorphisms which are commutative with every operator of $H$, there results a group of order $2 h^{2}$ which satisfies the conditions in question.

3. Number of Conjugates Equal to the Order of the Commutator Subgroup Diminished by One. The general problem of determining all the groups which satisfy the conditions expressed in the heading of the present paper seems to be quite difficult. There are, however, various special cases which may possibly contribute towards this general solution and which lead to interesting results. Among these is the case when the group $G$ contains a set of conjugate operators whose number is one less than the order $k$ of its commutator subgroup $K$, in addition to containing a set whose number is equal to $k$. It is easy to see that the former condition implies the latter while the converse is evidently not true.

In fact, if $G$ involves a set of $k-1$ conjugates, the remaining 
operator in the co-set with respect to $K$ to which these conjugates belong must be an invariant operator. Hence $K$ must involve $k-1$ conjugates under $G$ and it must therefore be an abelian prime power group of type $(1,1,1, \cdots)$. Since the operators of $K$ are transformed according to a transitive substitution group of degree $k-1$ under $G$, there must be an operator of $G$ which transforms them according to a substitution of degree $k-1$. As this operator is commutative with only the identity in $K$ it has $k$ conjugates under $G$. Hence the following theorem has been established:

THEOREM. If a group contains a set of conjugate operators whose number is equal to the order of the commutator subgroup diminished by one, it must also contain a set of conjugate operators whose number is equal to the order of its commutator subgroup, and this subgroup must be an abelian prime power group of type $(1,1,1, \cdots)$.

No co-set of $G$ with respect to $K$ involves more than one invariant operator, and when it involves one such its remaining operators constitute a single set of conjugates under $G$. The invariant operators in all of these co-sets constitute the central of $G$ and this has only the identity in common with $K$. Hence $G$ involves the abelian group which is the direct product of $K$ and the central of $G$. Each of the operators of $G$ which does not appear in this direct product has $k$ conjugates under $G$, and the order of $G$ is equal to $k$ times the order of this direct product since the $k-1$ operators of prime order contained in $K$ are transformed under $G$ according to a regular group. The fact that this group is regular results directly from the facts that it is transitive and abelian.

Whenever a group contains a commutator subgroup of prime order $p$ it must also contain at least one set of $p$ conjugates, but when the commutator subgroup is neither of prime order nor the identity the number of operators in a set of conjugates is not necessarily equal to the order of the commutator subgroup. If a group whose commutator subgroup is of order $p$ contains also sets of conjugates involving more than one but less than $p$ operators, each of its co-sets with 
respect to $K$ which involves less than $p$ conjugates involves just one invariant operator. The other operators of such a co-set are conjugate in sets of $k$, where $k$ is a divisor of $p-1$ and has the same value for all such sets.

The fact that the commutator subgroup of $G$ must be abelian whenever $G$ contains a set of conjugate operators whose number is one less than the order of its commutator subgroup may also be regarded as a special case of the theorem that in any group whatever, the difference between the order of the commutator subgroup and the number of operators in a complete set of conjugates is a multiple of the number of the conjugates of any one of the operators of this set under the commutator subgroup. In particular, when there is a set of $k-1$ conjugate operators they must be commutative with every operator of $K$ and hence $K$ must be abelian.

4. The Number of Conjugates of Every Non-Invariant Operator is Equal to the Order of the Commutator Subgroup. When the number of operators in every complete set of non-invariant conjugates of the group $G$ is equal to $k$, the order of its commutator subgroup $K$, it follows directly that $K$ must be in the central of $G$ since none of its operators could have $k$ conjugates under $G$. To prove that $K$ must be a prime power group it is only necessary to note that if $s$ is any non-invariant operator of $G$ then the operators of $G$ which are commutative with $s$ constitute an invariant subgroup of index $k$ under $G$, and the corresponding quotient group is simply isomorphic with $K$ since $s$ transforms the operators of $G$ into themselves multiplied by the various operators of $K$.

If the order of $K$ is not of the form $p^{m}, p$ being a prime number, let $q$ and $p$ be two different prime divisors of $k$. To an operator of order $q$ in the given quotient group there must correspond an operator of order $q^{\beta}$ in $G$. As this operator would have $k$ conjugates under $G$ it would have to be transformed under $G$ into itself multiplied by an operator of order $p$ contained in the central of $G$. This is evidently impossible since the order of this product could not be of the form $q^{\beta}$ Hence we have established the following theorem : 
THeOREM. If the number of operators in every set of noninvariant conjugates of a group is equal to the order of the commutator subgroup, then the order of this subgroup is of the form $p^{m}, p$ being a prime number.

From the preceding theorem it results directly that $G$ is the direct product of an abelian group and a non-abelian group whose order is of the form $p^{m}$. It is easy to prove that $K$ must be of type $(1,1,1, \cdots)$. If this were not the case the operator $s$ of $G$ which was defined above would transform an operator $t_{1}$ into itself multiplied by an operator of order $p^{\alpha}$ while it would transform $t_{2}$ into itself multiplied by an operator of order $p^{\alpha+1}$ whose $p$ th power is the multiplier of $t_{1}$ under $s$, $p^{\alpha+1}$ being an operator of highest order contained in $K$. Hence it may be assumed that $t_{2}{ }^{p}=t_{1}$. This is impossible since $G$ would also have to contain an operator which would transform $t_{1}$ into itself multiplied by an operator of order $p^{\alpha+1}$. This proves also incidentally that the $p$ th power of every operator of $G$ is found in the central of $G$.

In the special case when $k=p$ it is easy to prove that the order of the central quotient group of the Sylow group $P$ of order $p^{m}$ would have for its order an even power of $p$. In fact, all the operators which are commutative with a noninvariant operator $s_{1}$ of $P$ constitute a subgroup of index $p$. If $s_{2}$ is any operator in $P$ which is not commutative with $s_{1}$, then the cross-cut of the subgroups composed of all the operators which are commutative with $s_{1}$ and $s_{2}$ respectively will be of index $p^{2}$ under $P$. This cross-cut includes the central of $P$ and if it includes other operators we may find a subgroup of index $p^{2}$ contained in it and involving the central of $P$. As this process may be continued until we arrive at the central the theorem in question has been proved.*

The University of Iluinors

* Cf. J. W. A. Young, American JournaL, vol. 15 (1893), p. 171. 